\title{
ARTICLE OPEN \\ Orbitofrontal cortex is selectively activated in a primate model of attentional bias to cocaine cues
}

\author{
Eunha Baeg ${ }^{1,3}$, Hank P. Jedema ${ }^{1,4}$ and Charles W. Bradberry ${ }^{1,2,4}$
}

\begin{abstract}
Attentional bias to drug-associated cues correlates with extent of current use, and risk of relapse among those attempting abstinence. Electroencephalogram (EEG) and functional imaging measures in clinical studies have previously investigated the neural basis of attentional bias, but the lack of animal models precluded investigation at the single-unit level. To complement results obtained from clinical studies, we have employed a non-human primate model of attentional bias to cocaine cues while simultaneously recording single-unit activity in cortical and striatal regions implicated in reward processing. Rhesus macaques conditioned to associate particular colors with cocaine or water reward performed an attentional bias task, in which those colors served as irrelevant distractors. Concurrently, multiple electrode arrays for recording single-unit activity were acutely implanted into the orbitofrontal cortex, anterior cingulate cortex, dorsal anterior striatum, and ventral striatum. As in clinical studies, attentional bias was indicated by elongated response times on trials with cocaine-associated distractors compared with trials with waterassociated, or control unconditioned distractors. In both animals studied, across an unbiased sample of neurons, the orbitofrontal cortex differentiated distractor condition by the proportion of single-units activated, as well as by population response. In one of the two, the anterior cingulate cortex did as well, but neither striatal region did in either animal. These direct measures of singleunit activity in a primate model complement clinical imaging observations suggesting that cortical mechanisms, especially in orbitofrontal cortex, are likely involved in attentional bias to cocaine-associated environmental cues.
\end{abstract}

Neuropsychopharmacology (2020) 45:675-682; https://doi.org/10.1038/s41386-019-0499-0

\section{INTRODUCTION}

Reward-driven behavioral disorders, such as drug abuse, impose a significant disease burden upon society. Driving the societal impact of drug use is relapse among those seeking to abstain, and extent of use among those who are not. Neural encoding of relapse can be difficult to study in animal models because it is a one-time event, followed by transition to the pharmacological context, thereby limiting the statistical power of neural recordings in the absence of drug. This is especially true for primate studies which use small numbers of subjects.

Attentional bias to drug cues is a phenomenon whose neural encoding can provide insight into mechanisms relevant to addiction. Across individuals, the strength of attentional bias toward drug-linked cues is strongly associated with relapse in abstinent users, as well as the quantity and frequency of current use in nontreatment seekers [1]. This relationship is particularly strong with psychostimulants such as cocaine [2]. Experimentally, bias is often measured as an increase in response times on a relatively simple task, during which drug, or nondrug cues are presented as irrelevant distractors [2]. Despite being widely investigated clinically, there are currently no preclinical models for studying it. This is a critical need, because the imaging and EEG approaches typically used in clinical studies do not provide signals unambiguously linked to actual "output" neural activity from a given region [3, 4]. Only invasive, single-unit electrophysiological studies can provide high temporal resolution of cocaine cue reactivity. Also, depending on task/acquisition parameters, attentional bias has been linked to both increased and decreased frontal activity in imaging studies [2]. We report herein a non-human primate model of attentional bias that is ideally suited for neural recordings in animals because measurements of bias entail repeated cue exposure without drug administration across multiple trials, thereby increasing statistical power.

While rhesus macaques performed the bias task, we conducted single-unit recordings in both cortical and subcortical regions involved in reward processing. We recorded in the orbitofrontal (OFC) and anterior cingulate (ACC) cortex, which clinical imaging studies have identified as especially responsive to drug cues and as sites of cognitive dysfunction associated with addiction [5, 6]. Two striatal regions frequently implicated in addiction were also examined. Ventral striatum (VST) shows increased metabolic activity during reward evaluation and decision-making tasks in humans [7], and in concert with the OFC, is believed to be a "final common output" for value-based decisions [8]. Dorsal striatum (DST) is also implicated in addiction, particularly as drug-use transitions from a goaloriented to a habitual behavior [9].

\footnotetext{
${ }^{1}$ Department of Psychiatry, University of Pittsburgh, Pittsburgh, PA, USA and ${ }^{2}$ VA Pittsburgh Health Services, Pittsburgh, PA, USA Correspondence: Charles W. Bradberry (charles.bradberry@nih.gov)

${ }^{3}$ Present address: Center for Neuroscience Imaging Research, Institute for Basic Science (IBS), Suwon 16419, Republic of Korea

${ }^{4}$ Present address: NIDA Intramural Research Program, 251 Bayview Blvd, Suite 200, Baltimore, MD 21224, USA
}

Received: 3 April 2019 Revised: 11 July 2019 Accepted: 18 July 2019

Published online: 28 August 2019 


\section{MATERIALS AND METHODS}

Subjects

Two adult rhesus monkeys (15-04, a female 13.3 years old at study end, and 19-04, a male 16.5 years old at study end, weighing 6.5 and $8.5 \mathrm{~kg}$, respectively) were used. Each had extensive experience with intravenous cocaine self-administration prior to conditioning of cues used in this study, making them ideal subjects to model long-term human use. Animal 15-04 had $701 \mathrm{mg} / \mathrm{kg}$ cocaine over 7 years and $120 \mathrm{mg} / \mathrm{kg}$ cocaethylene (only in the first 2 years) prior to the first electrophysiological recording session for the data reported herein. The data from 67 sessions over 6 months were used, during which time an additional $59 \mathrm{mg} / \mathrm{kg}$ cocaine was selfadministered. Animal $19-04$ had $553 \mathrm{mg} / \mathrm{kg}$ cocaine over 8 years, and $13 \mathrm{mg} / \mathrm{kg}$ cocaethylene (only in the first 2 years) prior to the initial electrophysiological recording session. The data from 104 sessions over 2 years were used, during which time an additional $189 \mathrm{mg} / \mathrm{kg}$ cocaine was self-administered. Animals were singly housed in a room with visual and auditory contact with conspecifics. They were chaired using pole and collar methods (Primate Products), and all behavioral and electrophysiological procedures were conducted in a sound-proofed audiometric booth (Eckel Industries, Morrisburg, Ontario), in which a touch screen monitor (Carroll Touch, ELO Touch Inc.) was fixed on one wall. Animals were water regulated, with daily supplementation provided after experimental sessions. E-prime software (Psychology Software Tools, Sharpsburg, PA) was used for stimulus presentation and response recording. All procedures used were approved by the University of Pittsburgh and Pittsburgh Veterans Administration Institutional Animal Care and Use Committees, and were in conformance with the Eighth edition of the NIH Guide for the Care and Use of Laboratory Animals.

\section{Attentional bias task}

The touch screen layout of the task is depicted in Fig. 1a. On each trial, one of six semi-randomly selected positions of the $2 \times 3$ array would turn black, and touching that target would result in water delivery $(0.07 \mathrm{ml} / \mathrm{kg})$ and initiation of a $3-5 \mathrm{~s}$ variable intertrial interval. Simultaneous with target onset, and irrelevant to the task requirements, one of the remaining five positions (location semirandomized) changed to a color associated either with intravenous cocaine availability (cocaine discriminative cue, CD) or water reward availability (water discriminative cue, WD), a color that accompanied cocaine infusion (cocaine Pavlovian cue, CP), or a control color (non-associated cue, NA) that had no conditioned significance. Thus, one of the four distractor types was always presented at the same time as the target whose touch produced an actual water reward. Within each session, each cocaine or water distractor was presented 40 times, and the non-associated distractor was presented 80 times. (The number of trials was limited because of the need for the animals to remain chaired following the bias task for the self-administration session needed to maintain saliency of the distractor cues.) Failure to respond, or touching outside the target was considered an error, and only accurate trials were used. Actual colors of distractor cues differed between animals, and corresponded to those used in selfadministration cue conditioning as described below. Colors used in the figures are for ease of visualization.

Self-administration cue conditioning

Following completion of the attentional bias task, animals performed an initial block of ten water trials followed by ten cocaine trials in the same chamber and using the same touch screen. Those self-administration sessions continued throughout the study in order to maintain the saliency of the cues, as it has been shown that attentional bias decreases with abstinence [10]. The physical layout of the cues and target were distinct from the bias task, as shown in Fig. 1b. The configuration was chosen to emulate a similar arrangement to the electromechanical apparatus with colored lights and a response lever that animals had originally been trained on, and which was previously described [11]. Water and cocaine trials were always presented in separate blocks, beginning with water. Color filled a pair of squares $30 \times 30$ $\mathrm{mm}$ (blue for animal 15-04, green for 19-04) to indicate water was available. Upon touching a centrally placed target $(60 \mathrm{~mm}$ wide $X$ $45 \mathrm{~mm}$ high) three times, $0.07 \mathrm{ml} / \mathrm{kg}$ of water was delivered via a sipper tube, the water discriminative cue was offset, and a $30 \mathrm{~s}$ intertrial interval began. After ten trials, the cocaine block of trials began. Up to ten infusions of cocaine $(0.1 \mathrm{mg} / \mathrm{kg}$ i.v.) were available, with an intertrial interval of $5 \mathrm{~min}$. On each trial, a $36 \times$ $36 \mathrm{~mm}$ cue to the left of midline (red for animal 15-04, orange for 19-04) indicated that upon touching the target either five (animal 15-04) or ten times (animal 19-04) the cocaine infusion would begin. At that point the discriminative cue was offset, and during the infusion, which took $18 \mathrm{~s}$ to complete, the $36 \times 36 \mathrm{~mm}$ cue to the right of midline (green for animal 15-04, blue for 19-04) was presented. During the intertrial intervals, all cues were off. Cocaine was delivered to the monkey via a vascular access port located mid-scapula from which a catheter was connected to either a jugular or femoral vein [12].

\section{Electrophysiological recording}

Animals were head bolted throughout recording procedures. Recording electrodes were placed using a centrally positioned circular chamber (Crist Instruments, Hagerstown, MD) overlying OFC, ACC, DST, and VST. Placements were calculated from coregistered $M R$ and computerized tomography $(\mathrm{CT}) \mathrm{X}$-ray images using the software "monkey cicerone" [13], which visualized the recording chamber in relation to the neuroanatomy. Activities of single units were recorded with acutely placed bundles of Teflon insulated tungsten microwires $(50 \mu \mathrm{m}$ diameter, California Fine Wire, Grover Beach, CA). Each bundle, consisting of four wires, was independently lowered through a $23 \mathrm{G}$ sharpened guide tube using an electronically controlled microdrive (Plexon, Dallas, TX) to one of the target regions. Six independently placed bundles were used in each session. There was no pre-screening for task selectivity of neurons. The standard procedure was to take $30 \mathrm{~min}$ to isolate units, followed by $30 \mathrm{~min}$ for stabilization prior to recording. Neural activity was recorded using a Plexon Multichannel Acquisition Processor system (Dallas, TX) and sorted using Offline Sorter (Plexon). Further processing was conducted using Neuroexplorer (Nex Technologies) and Matlab (MathWorks Inc.).

\section{Data analysis and statistics}

Because mean response times were not normally distributed, we compared across distractor condition with a Friedman Repeated Measures Analysis of Variance on Ranks, followed by Tukey's all pairwise comparison post hoc test. For analysis of firing rate at the population and single-unit levels, Bonferroni-corrected paired $t$ tests were used as described in the Results section. For trial by trial regression of firing rate with response time, a pretrial baseline period of $0.5 \mathrm{~s}$ was used, and the change in firing rate was calculated for duration from target onset to time of response. Session by session regression of firing rate and response time was done by computing an average change in firing rate over the entire session and average response time for the entire session, using the time windows as for trial by trial. All trial types were included in both approaches. Corrections for multiple comparisons are indicated in the Results section.

\section{RESULTS}

Animals show attentional bias to cocaine cues

Consistent with the simplicity of the task, accuracy (successful touching of the black target resulting in water reward) was high (Fig. 1c), with no differences across distractor condition. However, as in clinical assessments, elongated response times on trials when 
A

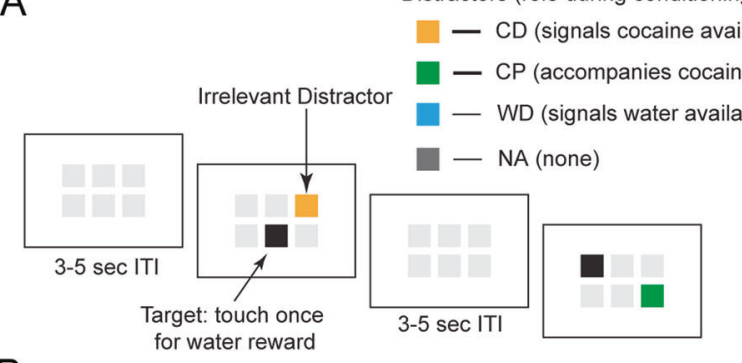

B

First block: $\square$ WD signals water availability

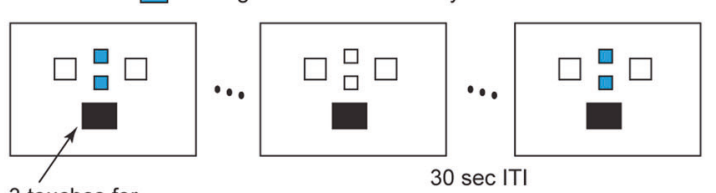

3 touches for

water reward

Second block: $\square$ CD signals cocaine availability

CP accompanies infusion

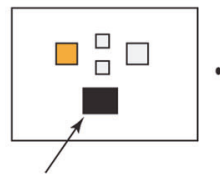

5 or 10 touches for cocaine infusion

C

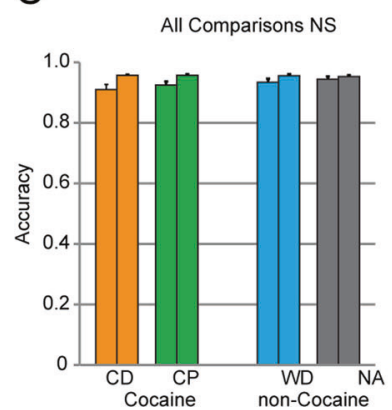

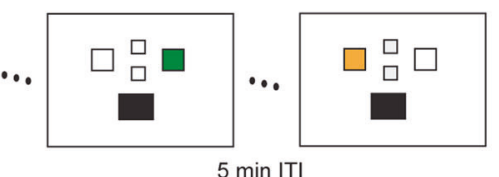

$18 \mathrm{sec}$ coc infusion

$(0.1 \mathrm{mg} / \mathrm{kg})$

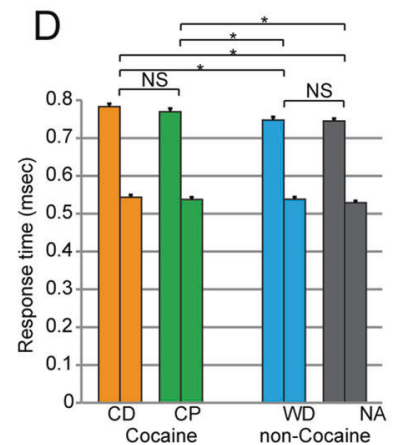

E

Animal 15-04 Animal 19-04
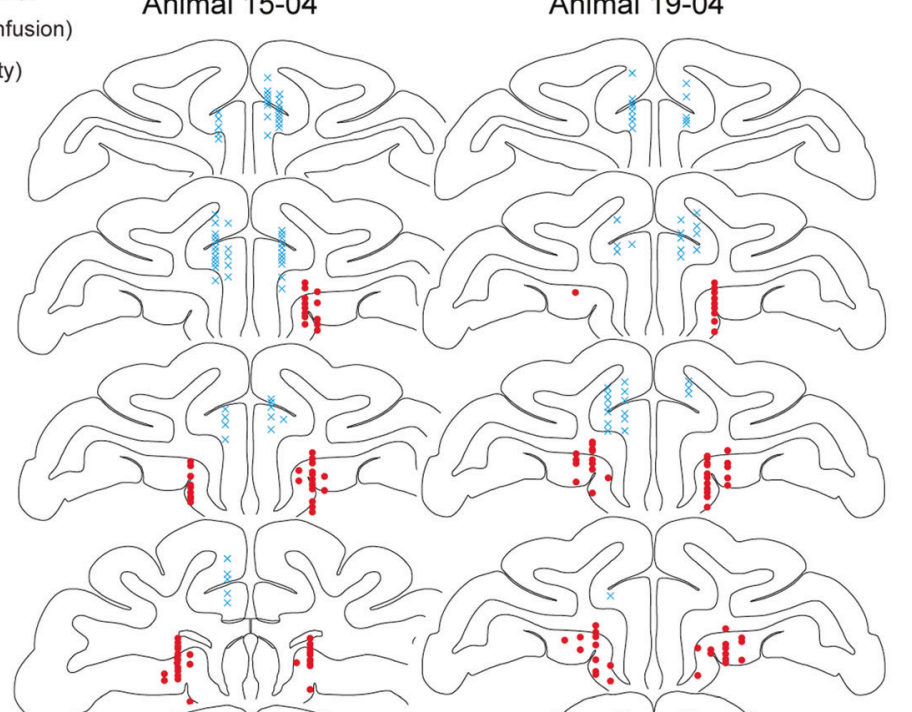

(
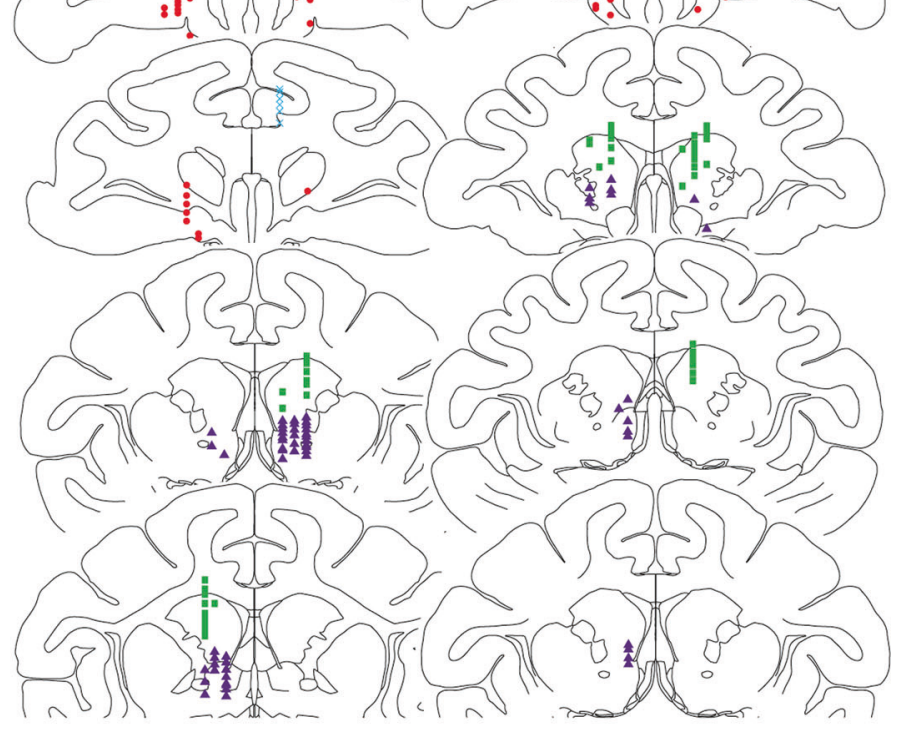

Fig. 1 Task structures, behavior, and anatomical placements. a Attentional bias task structure. Touching the black target resulted in water reward. Distractors appeared simultaneously with target, but were irrelevant to task contingency. Locations of target and distractor were pseudo random on each trial. b Cue conditioning (self-administration) task structure. First block provided ten water reward $(0.07 \mathrm{ml} / \mathrm{kg}$ ) trials, second block presented 10 i.v. cocaine reward $(0.1 \mathrm{mg} / \mathrm{kg})$ trials. c Bias task accuracy. Animal 15-04 is shown in the left bar for each condition, and 19-04 is shown in the right bar. Comparisons are for both animals combined. Accuracy did not differ by distractor condition. $\mathbf{d}$ Bias task response times. Cocaine-associated distractors were associated with elongated response times compared with water and non-associated distractors, ${ }^{*} p<0.05$. e Summary of electrode placements for each animal. o, OFC; $x, A C C ; \Delta$, VST; $\square$, DST. Depths of electrode placements were calculated based on the position of the cranial cylinder in co-registered MR and CT images

the task-irrelevant distractor was drug associated indicated attentional bias (Fig. 1d) [1, 2, 14]. Collapsed across both animals, chi-square was 75.6 with $\mathrm{df}=3: p<0.001$ for main effect of distractor type. When the distractor cue color matched either the $\mathrm{CD}$ or $\mathrm{CP}$ cue, response times were significantly longer than when the distractor cue color matched the NA or WD cue. (Tukey's all pairwise comparison post hoc: $C D$ vs NA, difference of ranks $=$ 191, $q=11.3, p<0.05 ; \mathrm{CD}$ vs WD, difference of ranks $=142, q=$ 8.41, $p<0.05$; CP vs NA, difference of ranks $=130, q=7.70, p<$ $0.05 ; \mathrm{CP}$ vs WD, difference of ranks $=81, q=4.80, p<0.05)$. Response times for trials with $C D$ and $C P$ did not differ from each other (difference of ranks $=61, q=3.61, p>0.05$ ), nor did response times for trials with the WD cue differ from trials with the NA cue (difference of ranks $=49, q=2.90, p>0.05$ ). Based on this pattern of response times, for subsequent analyses, the four distractor conditions were collapsed to two for greater statistical power: cocaine associated, and non-cocaine. These above results are for both animals combined. Comparisons within each individual animal followed the same pattern except that for animal 19-04, response times with CP did not differ from distractor WD (difference of ranks $=26, q=1.98, p>0.05$ ) and WD differed from NA (difference of ranks $=52, q=3.95, p<0.05$ ).

Single-unit recording during bias task

Simultaneous recordings were made in OFC, ACC, DST, and VST. Figure $1 \mathrm{e}$ is a compilation of anatomical placements in both animals. Based on demarcations in the Paxinos rhesus atlas [15] OFC placements were in regions $13 \mathrm{~b}$ and $13 \mathrm{~m}$ [16]. In ACC, placements were in regions 9/32, 32, 24b, and 24c. All striatal placements were pre-commissural, with what we have termed DST being in the head of the caudate. What we term VST matches that described by Haber et al. [17]. 
Table. 1. Summary statistics for single unit recordings. FR is firing rate, SEM is standard error of the mean, OFC is orbitofrontal cortex, ACC is anterior cingulate cortex, DST is dorsal striatum, VST is ventral striatum

\begin{tabular}{lllll}
\hline & OFC & ACC & DST & VST \\
\hline Both Animals Combined & & & & \\
\# Units & 503 & 412 & 196 & 166 \\
\# Sessions & 146 & 114 & 100 & 76 \\
Units/session & 3.45 & 3.61 & 1.96 & 2.18 \\
Mean FR (Hz) +/- SEM & $6.49+/-0.23$ & $4.99+/-0.21$ & $4.45+/-0.23$ & $7.18+/-0.57$ \\
Animal 15-04 & & & & \\
\# Units & 191 & 116 & 61 & 67 \\
\# Sessions & 59 & 43 & 35 & 37 \\
Units/session & 3.24 & 2.70 & 1.74 & 1.81 \\
Mean FR (Hz) +/- SEM & $6.77+/-0.47$ & $4.61+/-0.33$ & $4.66+/-0.43$ & $9.32+/-1.06$ \\
Animal 19-04 & & & & \\
\# Units & 312 & 296 & 135 & 99 \\
\# Sessions & 87 & 71 & 65 & 39 \\
Units/session & 3.59 & 4.17 & 2.08 & 2.54 \\
Mean FR (Hz) +/- SEM & $6.33+/-0.24$ & $5.13+/-0.26$ & $4.07+/-0.28$ & $5.73+/-0.58$ \\
\hline
\end{tabular}

Combined, there were a total 171 sessions (67 from 15-04, and 104 from 19-04). Table 1 provides a breakdown by region of the total number of units, the number of sessions in which a unit was recorded, the number of units per session, and the mean baseline firing rates. A minimum firing rate of $1.0 \mathrm{~Hz}$ was used to select isolated neurons for analysis in order to permit a determination of baseline firing rate. This yielded, for 15-04, 6.5+/- 0.40 (SEM) units (from all regions combined) per recording session (for all units isolated, $8.4+$ / - 0.46). For 19-04, $8.6+$ +/ 0.54 (SEM) units/ session $(>1 \mathrm{~Hz}$ ) were obtained (all isolated units, $11.4+/-0.73$ units/session). Table S1 provides a breakdown of \# successful trials by distractor cue type for each animal. Figure $\mathrm{S} 1$ shows histograms of the distribution of baseline firing rates across each region.

Figure 2a shows peri-event raster plots and mean firing rate curves for eight different OFC single units across the two animals, and with varying baseline firing rates. Comparison of the responses to the cocaine (combined $C D$ and $C P$ ) and noncocaine (combined WD and NA) distractor trials was done by paired $t$ test, in which the mean firing rate of the neuron was compared across conditions using a sliding $200 \mathrm{msec}$ window stepped at $20 \mathrm{msec}$ intervals from $0.2 \mathrm{~s}$ prior to target/distractor presentation (the first window is from 0.4 to $0.2 \mathrm{~s}$ prior) to $0.7 \mathrm{~s}$ after. A given window was considered significant if $p<0.0011$ (Bonferroni-corrected value $0.05 / 46$ windows), and a red dot was placed at the end of that window.

We determined if there were regional differences in the pattern of encoding of distractor identity at the single-unit level. Because we employed a non-biased analysis of all units with $1 \mathrm{~Hz}$ or greater baseline firing rate, our approach evaluated differences in the proportion of all units that significantly responded to the $C D$ and WD distractors. (Only these two cues were used because the number of trials with the NA cue differed from the other conditions.) The number of spikes during the interval from 50 to $250 \mathrm{msec}$ following cue onset was compared with the same length interval just prior to cue onset ( $p<0.05$, paired $t$ test). This short latency window was chosen in order to focus on more automatic early stages of processing likely to underlie attentional capture by reward-associated cues $[18,19]$. The proportion of individual units that changed firing rate was then compared across the two distractor conditions for each region by chi-square test. As can be seen in Fig. 2b, only in the OFC in both animals did the proportion of responsive units differ across distractor condition. The increased firing upon cocaine cue presentation is short-lived. Comparing firing rates upon reward delivery after
CD and WD cues showed there was no difference in any region (Fig. S2).

Regionally selective encoding of the cocaine-associated distractor was also seen at the population level. The same sliding window analysis used with the single units indicated that in both animals (and their combined data sets), the OFC discriminated between distractor conditions at the population level (Fig. 3a). To ensure that we are adequately correcting for multiple comparisons, a baseline period from -1.0 to $-0.8 \mathrm{~s}$ prior to cue onset was also compared and as can be seen in Fig S3, no signficant windows were detected in either animal individually, or combined. In one animal, the ACC also discriminated the distractor conditions, but in the other animal did not. Therefore, given that this was not observed in the unbiased total pool of neurons in both animals, we discount the generality of that observation. As a further characterization of the population response, we divided up all analyzed units into those with increased or decreased firing upon cue presentation. Significant encoding in the cortical regions was only seen among neurons which increased in firing, and interestingly, we also saw a brief period of encoding among the excited units in ACC in the second animal as well. (Fig. S4). In animal 15-04, there were some ACC placements more caudal than those in 19-04. Figure S5A shows that limiting the ACC placements in 15-04 to more rostral ones did not alter the magnitude or timing of the differentiation.

In contrast to the cortical encoding of distractor condition, neither striatal site differentiated the conditions. To ensure that this result was not biased by the smaller number of units in the striatal sites, neurons from the cortical sites were shuffled, and the same number as in the VST (166) were randomly selected and the population response comparison was repeated. If the shuffled group showed a significant $p$-value (paired $t$ test, $p<0.0011$ ) at any time point, it was counted as a significant shuffle. The procedure was repeated 1000 times, and significant windows were found 996 times for OFC, 787 times for ACC, and 295 times for DST. Limiting our analysis to only the most medial placements in the VST (Fig. S5B), or the most lateral in DST (Fig. S5C) did not alter the pattern of results. Thus, at the population level, the OFC most selectively encoded distractor identity. ACC did so in one of the two animals, and no evidence of encoding was seen in either striatal region.

Roesch and Olson (supplement to ref. [20]) observed that OFC neurons are significantly more responsive to reward stimuli when presented contralateral to the recording site hemisphere. In our bias task, the $2 \times 3$ array of potential distractor locations were divided into contralateral, central, and ipsilateral locations, relative to the hemisphere of a recorded unit. As shown in Fig. 3b, there was a significant impact of distractor location such that when there was a cocaine-associated distractor present, the population response was greatest when it was contralateral, intermediate when central, and smallest when ipsilaterally located. Non-cocaine distractors showed no spatial encoding. In one of the two animals, the target (black square in Fig. 1), the only stimulus to actually produce a reward, showed this pattern of contralateral hemifield selectivity, but only when the distractor was not associated with cocaine. If a cocaine distractor was present, the spatial selectivity for the target was eliminated (data not shown).

Given that the mean firing rate of OFC neurons was higher and response times were longer on trials when a cocaine distractor was present, we examined the relationship between response time and firing rate in two ways. One was a trial by trial comparison regressing the change in firing rate from each trial's baseline with the response time on that trial, with all correct trial types included. The correlation coefficients for each neuron were then averaged (Fig. 4a). A positive value would be consistent with increased OFC firing "driving" elongated response times. This was not seen in either animal. In one animal, the mean values in each region did not differ from zero, while in the other, the slightly 
A
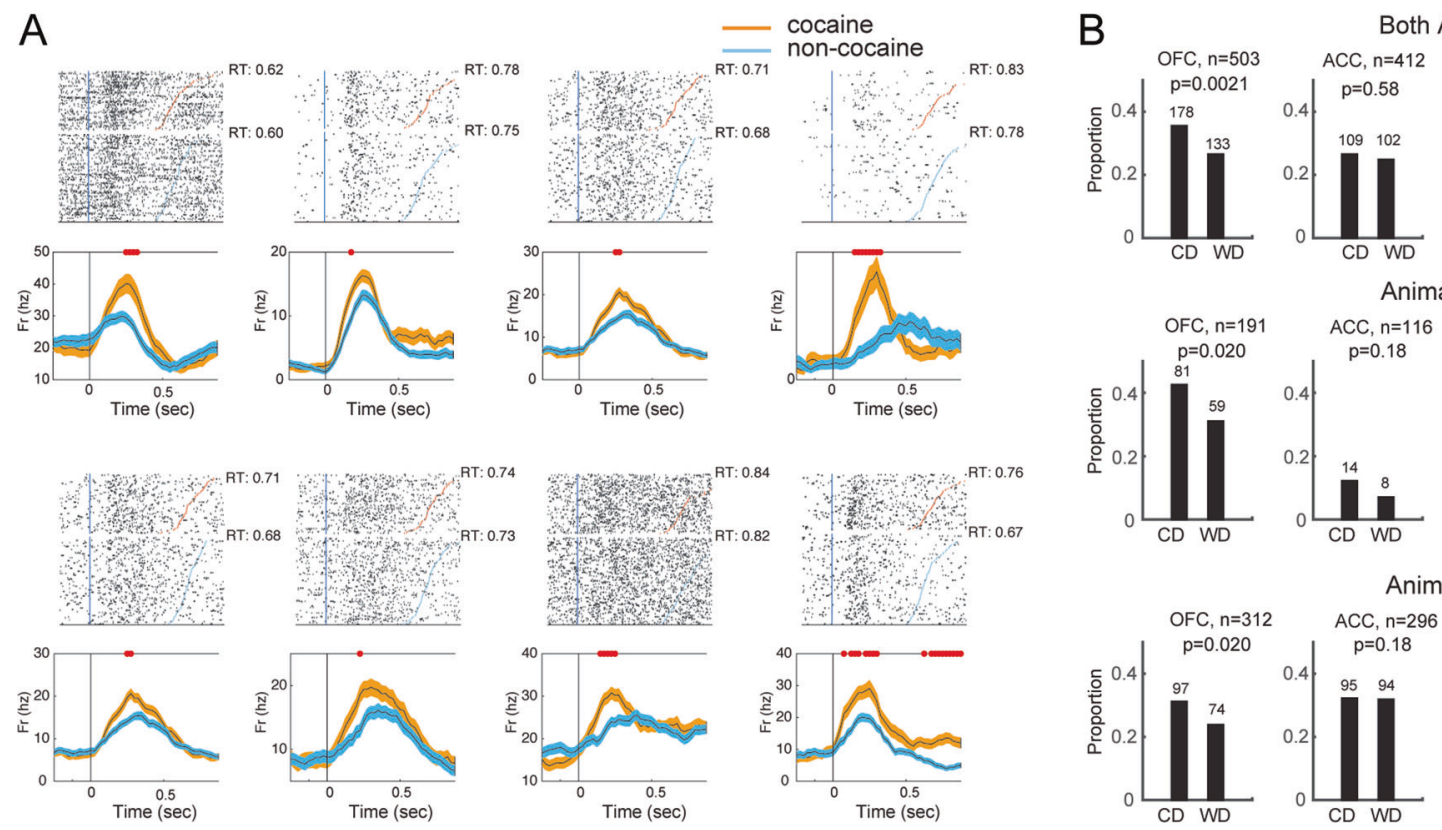

Both Animals
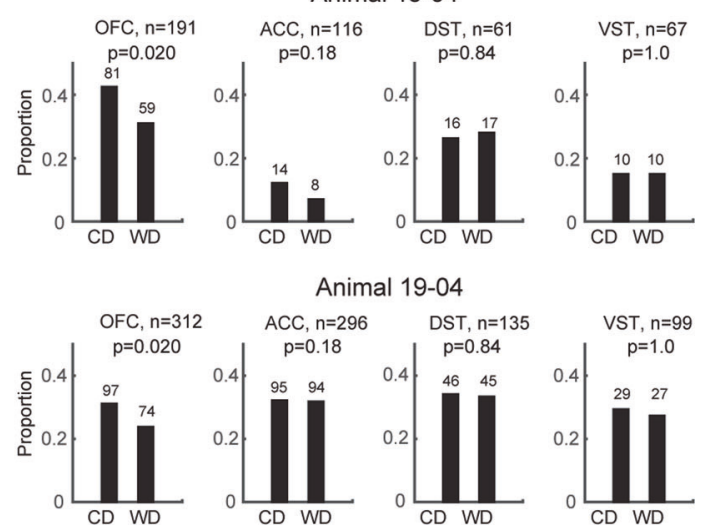

Fig. 2 Neural encoding of cocaine-associated distractors: single units. a Representative cue encoding neurons from OFC. Top shows rasters for cocaine and non-cocaine trials, with the response time marked in color. Within each cue type, trials are arranged in order of decreasing response time, with mean response time for each set of trials indicated. Peri-event histogram for each neuron is shown below the raster plot (mean $+/-$ SEM). Red dots are placed at the end of $200 \mathrm{msec}$ windows wherein response differs by paired $t$ test $(p<0.0011$, Bonferroni correction $p=0.05 / 46$ windows). $\mathbf{b}$ The proportion of single units whose firing rate changed following cue onset differed (by chi-square) for the CD and WD conditions only in OFC. The $200 \mathrm{msec}$ pre-cue baseline period was compared to the period 50-250 msec afer cue onset. Only the $C D$ and WD conditions were compared in order to make the number of trials in each condition equivalent. $N$ indicates the total number of neurons, and the number above each bar indicates the number (out of $n$ ) that changed firing rate from baseline

negative values were significantly different from zero. We also examined the relationship between OFC firing and response time at a session level by comparing the mean change in firing rate with the mean response time. Here, using all trial types, we did see a significant positive slope in the regression in both animals, and only in the OFC (Fig. 4b). This suggests that from session to session, there was variability in responsiveness of OFC units that significantly correlated with variability in response times, potentially suggesting the state of responsiveness of the animal to cues varied from day to day such that higher OFC firing was associated with longer response times.

\section{DISCUSSION}

This is the first animal model of attentional bias to drug cues we are aware of, and similar to clinical observations, elongated response times were observed when irrelevant drug-associated distractors were present during a relatively simple task. We examined single-unit activity during the task in four rewardprocessing regions, and only the OFC consistently encoded the cocaine-associated identity of task-irrelevant distractors in both animals. It is important to note that this is not a population study. Our observations only reflect patterns of neural encoding in two animals with a long history of cocaine self-administration. As is typical of all non-human primate electrophysiological approaches, statistical power is achieved through repetition within individual animals, and generality of the observation is inferred from consistency of effects across individual animals.

While many clinical studies of attentional bias toward drugassociated cues have focused on associations between bias and behavioral measures of craving, relapse, and current use, studies of the neural systems mediating their ability to capture attention are sparse. In general, there is strong evidence that cocaine cues engage cortical activity, particularly in OFC and ACC, which often show greater reactivity to drug cues than the striatum $[6,11,21-25]$. Neurons in OFC encode reward valuation $[20,26,27]$, they do so prior to an actual decision between options [27], their encoding of value-driven decisions is more stimulus-guided than action-guided [28], and they encode reward opportunities extremely rapidly $[11,29,30]$, consistent with automatic processing that is also characteristic of attentional bias [19]. Also of relevance to this report is recent work indicating that with covert shifts in attention, OFC units encode the value of the attended stimulus [31].

MR imaging studies during attentional bias tasks in cocaine users suggest an involvement of the OFC. Using a cocaine Stroop task, it was found that non-dependent recreational cocaine users, who showed no attentional bias, exhibited a hypoactivation in OFC relative to dependent users, when contrasting drug and control words [32]. In contrast to those group differences in OFC, no differences were observed between control and cocaine-linked words in any striatal region. Using a different color/drug word Stroop task and imaging parameters, Goldstein et al. showed that across individuals, the difference in accuracy between drug and neutral words correlated with the difference in BOLD signal between those conditions in the OFC, such that greater hypoactivations were associated with increased errors. This was interpreted to mean that greater distraction by drug words (increased errors) required greater OFC suppression of an emotional response [33]. Attentional bias in smokers has also been linked to increased BOLD response in ACC, anterior insula, and OFC Area 13 [34, 35].

Given the greater temporal resolution of unit recording, and that the link between altered regional output (pyramidal) unit activity and BOLD remains unclear [4], our results are valuable because they clearly indicate that OFC output is uniquely engaged when comparing the four regions examined. Only in the OFC was there observed a consistent encoding of cocaine-associated 

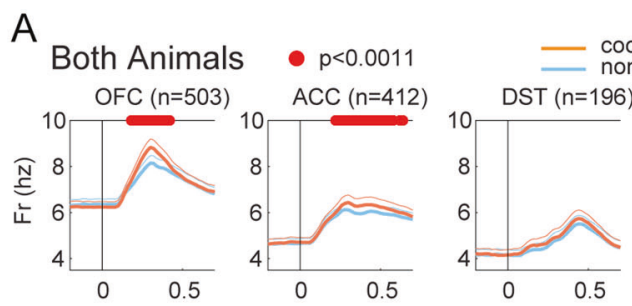

cocaine

Animal $15-04$
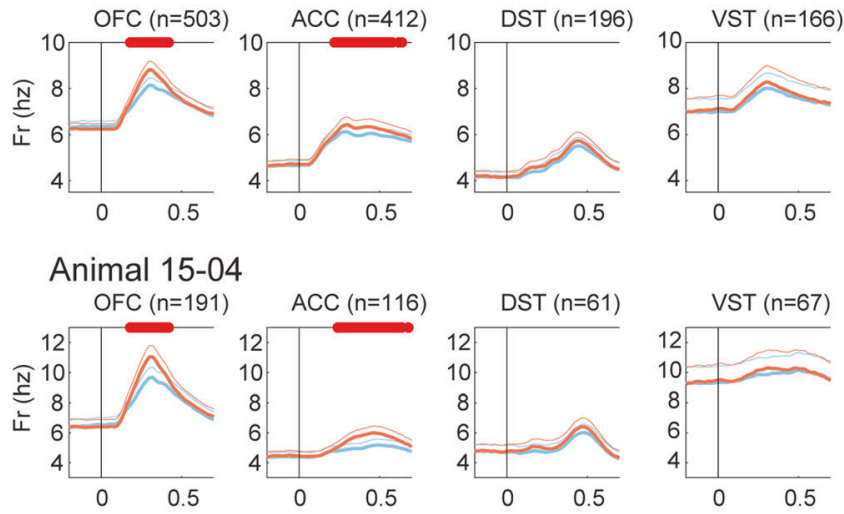

Animal 19-04
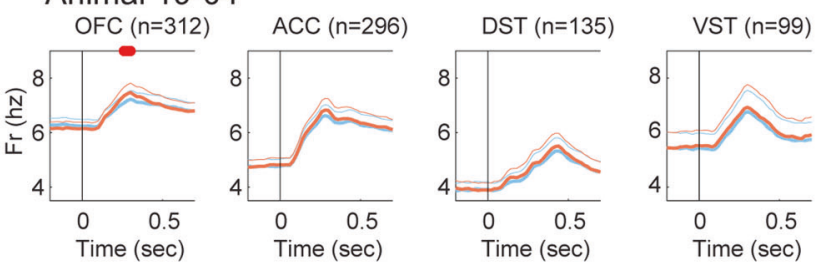

B
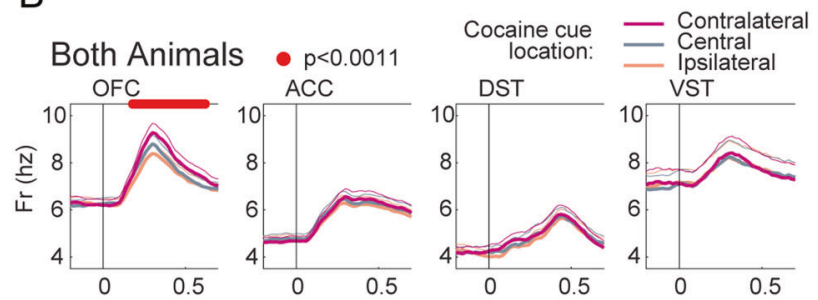

\section{Animal 15-04}
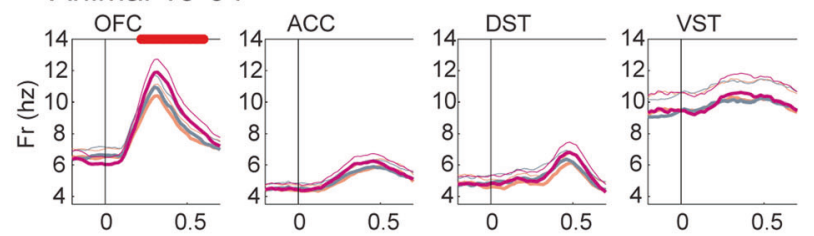

Animal 19-04
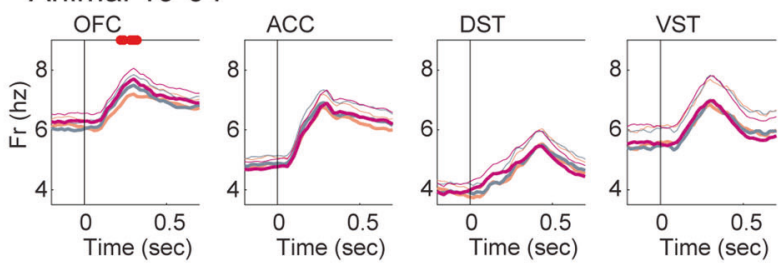

Fig. 3 Neural encoding of cocaine-associated distractors: population response. a Mean population response in each region (+ SEM) during attentional bias task, showing the significant encoding by OFC in both animals combined, as well as each separately. Red dots are placed at the end of $200 \mathrm{msec}$ windows (stepped at $20 \mathrm{msec}$ ), wherein response differs by paired $t$ test (Bonferroni-corrected $p<0.0011$ ). b Spatial encoding of distractor location in OFC. For each panel, the $2 \times 3$ array shown in Fig. 1 was divided into three by combining the upper and lower positions that were contralateral, ipsilateral, or central, relative to the hemisphere being recorded from. When the distractor is associated with cocaine, there is a significant contralateral hemifield selectivity. Red dots signify end of 200 msec windows, wherein the response differs by paired $t$ test (Bonferroni-corrected $p<0.0011$ ), thin line is + SEM

distractors both at a single unit, and at a population level of analysis. Another intriguing indication of the ability of cocaine distractors to selectively engage the OFC was the emergence of hemifield spatial selectivity only for them. There are conflicting reports as to spatial encoding by OFC [27, 29, 36-38]. A key difference between our approach and most others is that no gaze fixation was enforced, and it has been reported that the value encoding in the OFC is affected by gaze location [39]. Roesch and Olson (see Supplementary Material for [20] observed that $11 \%$ of OFC neurons responded more for reward-predicting stimuli in the hemisphere contralateral to the recording site than ispsilateral. Grattan and Glimcher [38] also observed that 10\% of OFC neurons showed hemifield selectivity. We observed a contralateral hemifield selectivity in OFC toward location of cocaine-associated distractors in both animals. No such selectivity for non-cocaine distractors was seen. There was a weak spatial selectivity for the target location in one animal when the distractor was not associated with cocaine, which was eliminated in the presence of a cocaine distractor. This is striking, given that the target is functionally more important: touching it produced an actual reward, as opposed to the distractor, which was irrelevant to the task contingencies.

A direct relationship between altered OFC firing and response times was not observed on a trial-by-trial basis. Given the small changes in response times that indicate attentional bias (for examples, see the mean response times for individual sessions on the single-unit raster plots in Fig. 2a), and the sparse output from OFC to motor control regions, this is not surprising, and there are no reports in the literature of trial-by-trial correlations of response latencies and OFC single-unit activity that we are aware of. The positive relationship between mean FR and response times across sessions was intriguing because it could imply that on some days, animals were more responsive to the behavioral effects of the cues than others.

In humans, stimuli repeatedly paired with higher value outcomes produce longer response times when presented as irrelevant distractors [40], similar to observations in attentional bias tasks. Imaging during this "reward-driven attentional capture" indicated a larger BOLD signal in the tail of the caudate on trials when high-value distractors were presented in the contralateral hemifield [41], matching observations that the tail of the caudate in the monkey contains neurons that preferentially encode contralaterally presented stimuli following repeated pairings with high-value outcomes even when the stimuli did not predict reward [42]. A group of presumed DA neurons in caudal lateral substantia nigra pars compacta that selectively project to the tail of the caudate also encoded stable value of stimuli outside of task contingencies, again, with a contralateral bias [43]. Given the similar impact on response times, differential encoding despite task irrelevance, and contralateral hemifield selectivity, it is intriguing to consider a link to our observations. PET imaging of dopamine D2 receptor ligand displacement indicated increased DA release broadly in anterior/posterior caudate and posterior putamen with response time differences associated with high value distractors [44]. However, those PET observations contrast with the MR imaging study [41] in that the response was not limited to the tail of the caudate. They also contrast with our single-unit measurements in homologous anterior caudate which revealed no encoding.

It has been widely hypothesized that attentional bias to drug cues is driven by phasic dopaminergic responses [1, 10, 18, 45], and drug-associated word exposure can increase BOLD signal in the mesencephalon [46]. Dopaminergic activation can result from positive reward prediction errors [47], but in a well-learned 
A Animal 15-04

$\mathrm{p}=0.0013$ OFC

$\mathrm{p}=0.0059 \mathrm{ACC}$

$\mathrm{p}=0.000015$ DST

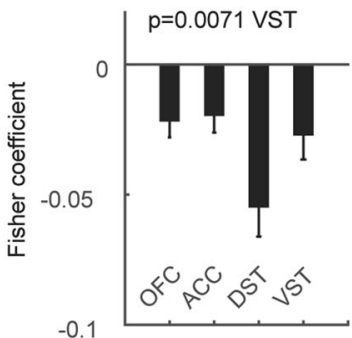

Animal 19-04

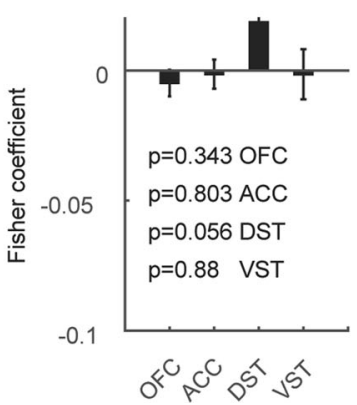

B Animal 15-04
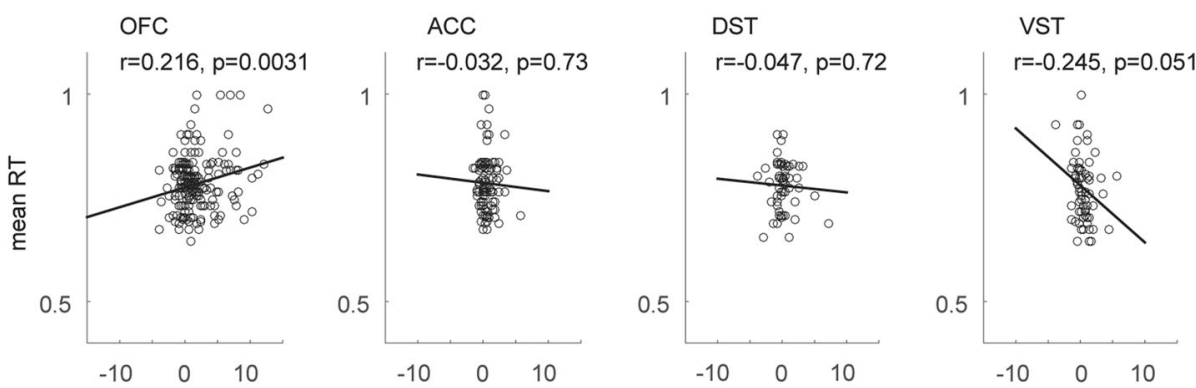

Animal 19-04
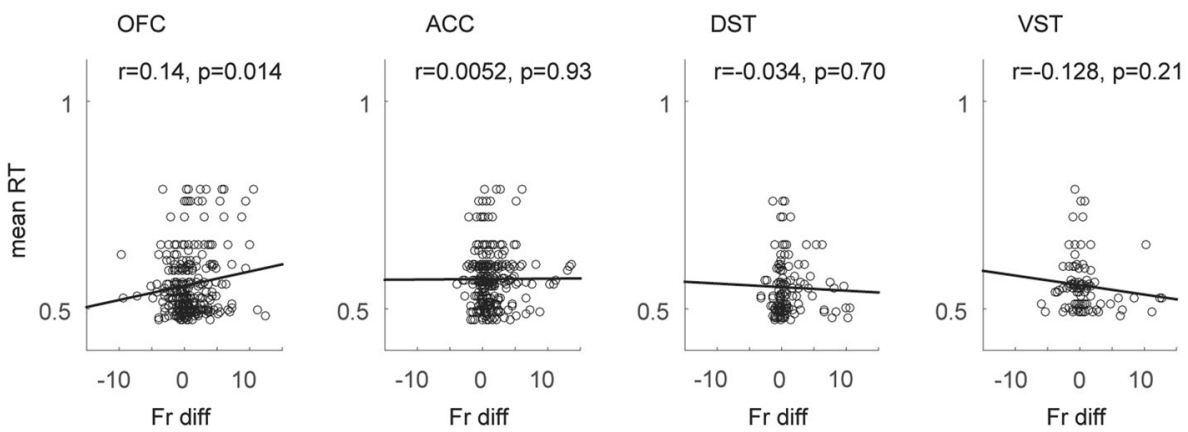

Fig. 4 Relationship of firing rate and response time. a For trial by trial analysis, the change in firing rate from baseline $(0.5 \mathrm{~s}$ prior to stimulus onset) was linearly regressed against the response time for that trial. Each resulting $R$-value was Fisher transformed, and the mean across all neurons was plotted in each region. The $p$-value is for comparison of the mean Fisher transformed $\mathrm{R}$ with zero. $\mathbf{b}$ The session by session relationship of firing rate with response time was determined by regressing the mean change from baseline for each neuron with the mean response time for that session. All correct trial types were used for both $\mathbf{a}$ and $\mathbf{b}$

task such as ours, irrelevant distractors offer no information as to immediate reward likelihood, and thus no differential encoding by DA neurons would be expected. Also, given the lack of DST or VST encoding of distractor identity, despite orders of magnitude greater innervation by DA relative to OFC or ACC [48], it could be argued that a dopaminergic basis is unlikely. We doubt the newly observed subset of DA neurons that innervate the tail of the caudate and stably encode value [43] could be driving the OFC responses we observed, because anatomical studies indicate no innervation of OFC from the region of substantia nigra containing those DA neurons [49]. However, definitive conclusions as to the role of those, or other dopaminergic neurons will require recordings of their activity during the attentional bias task.

This report is the first we are aware of, in which a preclinical model of attentional bias has been established for investigating potential mediation by distinct cortical and striatal regions known to be engaged in reward and affective processing. We observed that only neuronal firing in OFC consistently encoded distractor identity in both monkeys. One of the two animals also showed encoding in the ACC, but neither showed encoding in the DST or VST. Future work will need to directly examine the potential involvement of dopaminergic and other circuits, and also determine if drug cues uniquely engage the OFC, or if it is merely reward efficacy driving the encoding of irrelevant distractors.

\section{FUNDING AND DISCLOSURE}

This work was supported by NIH grant DA025636, VA Merit Review grant 1 I01 BX000782, the NIH/NIDA Intramural Research Program, and from the Republic of Korea, grant IBS-R015-D1. The authors declare no competing interests.

\section{ACKNOWLEDGEMENTS}

Excellent technical assistance by Kate Gurnsey, Jess Nicolazzo, and Carol Ehnerd is gratefully appreciated.

\section{AUTHOR CONTRIBUTIONS}

$E B$ and CWB designed the study, EB collected the data and was primary analyzer of the data, assisted by HPJ. EB and CWB were primary authors, assisted by HPJ.

\section{ADDITIONAL INFORMATION}

Supplementary Information accompanies this paper at (https://doi.org/10.1038/ s41386-019-0499-0).

Publisher's note Springer Nature remains neutral with regard to jurisdictional claims in published maps and institutional affiliations.

\section{REFERENCES}

1. Field M, Cox WM. Attentional bias in addictive behaviors: a review of its development, causes, and consequences. Drug Alcohol Depend. 2008;97:1-20.

2. Leeman RF, Robinson CD, Waters AJ, Sofuoglu M. A critical review of the literature on attentional bias in cocaine use disorder and suggestions for future research. Exp Clin Psychopharmacol. 2014;22:469-83.

3. Logothetis NK. What we can do and what we cannot do with fMRI. Nature. 2008;453:869-78.

4. Kim SG, Ogawa S. Biophysical and physiological origins of blood oxygenation level-dependent fMRI signals. J Cereb Blood Flow Metab. 2012;32:1188-206.

5. Schoenbaum G, Shaham Y. The role of orbitofrontal cortex in drug addiction: a review of preclinical studies. Biol Psychiatry. 2008;63:256-62.

6. Goldstein RZ, Volkow ND. Dysfunction of the prefrontal cortex in addiction: neuroimaging findings and clinical implications. Nat Rev Neurosci. 2011;12:652-69. 
7. Bartra O, McGuire JT, Kable JW. The valuation system: a coordinate-based metaanalysis of BOLD fMRI experiments examining neural correlates of subjective value. Neuroimage. 2013;76:412-27.

8. Kable JW, Glimcher PW. The neurobiology of decision: consensus and controversy. Neuron. 2009;63:733-45.

9. Everitt BJ, Belin D, Economidou D, Pelloux Y, Dalley JW, Robbins TW. Review. Neural mechanisms underlying the vulnerability to develop compulsive drugseeking habits and addiction. Philos Trans R Soc Lond B Biol Sci. 2008;363: 3125-35.

10. Parvaz MA, Moeller SJ, Malaker P, Sinha R, Alia-Klein N, Goldstein RZ. Abstinence reverses EEG-indexed attention bias between drug-related and pleasant stimuli in cocaine-addicted individuals. J Psychiatry Neurosci. 2017;42:78-86.

11. Baeg EH, Jackson ME, Jedema HP, Bradberry CW. Orbitofrontal and anterior cingulate cortex neurons selectively process cocaine-associated environmental cues in the rhesus monkey. J Neurosci. 2009;29:11619-27.

12. Bradberry CW, Barrett-Larimore RL, Jatlow $P$, Rubino SR. Impact of self-administered cocaine and cocaine cues on extracellular dopamine in mesolimbic and sensorimotor striatum in rhesus monkeys. J Neurosci. 2000;20:3874-83.

13. Miocinovic S, Noecker AM, Maks CB, Butson CR, McIntyre CC. Cicerone: stereotactic neurophysiological recording and deep brain stimulation electrode placement software system. Acta Neurochir Suppl. 2007;97(Pt 2):561-7.

14. Ersche KD, Bullmore ET, Craig KJ, Shabbir SS, Abbott S, Muller U, et al. Influence of compulsivity of drug abuse on dopaminergic modulation of attentional bias in stimulant dependence. Arch Gen Psychiatry. 2010;67:632-44.

15. Paxinos G, Huang X-F, Toga AW. The rhesus monkey brain in stereotaxic coordinates. San Diego: Academic Press; 2000.

16. Carmichael ST, Price JL. Architectonic subdivision of the orbital and medial prefrontal cortex in the macaque monkey. J Comp Neurol. 1994;346:366-402.

17. Haber SN, Knutson B. The reward circuit: linking primate anatomy and human imaging. Neuropsychopharmacol. 2010;35:4-26.

18. Chelazzi L, Perlato A, Santandrea E, Della Libera C. Rewards teach visual selective attention. Vis Res. 2013;85:58-72.

19. Awh E, Belopolsky AV, Theeuwes J. Top-down versus bottom-up attentional control: a failed theoretical dichotomy. Trends Cogn Sci. 2012;16:437-43.

20. Roesch MR, Olson CR. Neuronal activity related to reward value and motivation in primate frontal cortex. Science. 2004;304:307-10.

21. Kosten TR, Scanley BE, Tucker KA, Oliveto A, Prince C, Sinha R, et al. Cue-induced brain activity changes and relapse in cocaine-dependent patients. Neuropsychopharmacology. 2006;31:644-50.

22. Garavan H, Pankiewicz J, Bloom A, Cho JK, Sperry L, Ross TJ, et al. Cue-induced cocaine craving: neuroanatomical specificity for drug users and drug stimuli. Am J Psychiatry. 2000;157:1789-98.

23. Maas LC, Lukas SE, Kaufman MJ, Weiss RD, Daniels SL, Rogers VW, et al. Functional magnetic resonance imaging of human brain activation during cue-induced cocaine craving. Am J Psychiatry. 1998;155:124-6.

24. Wexler BE, Gottschalk CH, Fulbright RK, Prohovnik I, Lacadie CM, Rounsaville BJ, et al. Functional magnetic resonance imaging of cocaine craving. Am J Psychiatry. 2001;158:86-95.

25. Grant S, London ED, Newlin DB, Villemagne VL, Liu X, Contoreggi C, et al. Activation of memory circuits during cue-elicited cocaine craving. Proc Natl Acad Sci USA. 1996:93:12040-5.

26. Rolls ET, Grabenhorst F. The orbitofrontal cortex and beyond: from affect to decision-making. Prog Neurobiol. 2008;86:216-44.

27. Padoa-Schioppa C. Neurobiology of economic choice: a good-based model. Annu Rev Neurosci. 2011:34:333-59.

28. Luk $\mathrm{CH}$, Wallis JD. Choice coding in frontal cortex during stimulus-guided or action-guided decision-making. J Neurosci. 2013;33:1864-71.

29. Kennerley SW, Wallis JD. Encoding of reward and space during a working memory task in the orbitofrontal cortex and anterior cingulate sulcus. J Neurophysiol. 2009;102:3352-64.

30. Rudebeck PH, Ripple JA, Mitz AR, Averbeck BB, Murray EA. Amygdala contributions to stimulus-reward encoding in the macaque medial and orbital frontal cortex during learning. J Neurosci. 2017;37:2186-202.
31. Xie $Y$, Nie $C$, Yang T. Covert shift of attention modulates the value encoding in the orbitofrontal cortex. eLife. 2018;7:e31507.

32. Smith DG, Simon Jones P, Bullmore ET, Robbins TW, Ersche KD. Enhanced orbitofrontal cortex function and lack of attentional bias to cocaine cues in recreational stimulant users. Biol Psychiatry. 2014;75:124-31.

33. Goldstein RZ, Tomasi D, Rajaram S, Cottone LA, Zhang L, Maloney T, et al. Role of the anterior cingulate and medial orbitofrontal cortex in processing drug cues in cocaine addiction. Neuroscience. 2007;144:1153-9.

34. Janes AC, Pizzagalli DA, Richardt S, Frederick Bde B, Holmes AJ, Sousa J, et al. Neural substrates of attentional bias for smoking-related cues: an FMRI study. Neuropsychopharmacology. 2010;35:2339-45.

35. Elton A, Chanon VW, Boettiger CA. Multivariate pattern analysis of the neural correlates of smoking cue attentional bias. Pharm Biochem Behav. 2019;180:1-10.

36. Yoo SBM, Sleezer BJ, Hayden BY. Robust encoding of spatial information in orbitofrontal cortex and striatum. J Cogn Neurosci. 2018;30:898-913.

37. Tremblay L, Schultz W. Relative reward preference in primate orbitofrontal cortex. Nature. 1999;398:704-8.

38. Grattan LE, Glimcher PW. Absence of spatial tuning in the orbitofrontal cortex. PLOS ONE. 2014;9:e112750.

39. McGinty VB, Rangel A, Newsome WT. Orbitofrontal cortex value signals depend on fixation location during free viewing. Neuron. 2016;90:1299-311.

40. Anderson BA, Laurent PA, Yantis S. Value-driven attentional capture. Proc Natl Acad Sci USA. 2011;108:10367-71.

41. Anderson BA, Laurent PA, Yantis S. Value-driven attentional priority signals in human basal ganglia and visual cortex. Brain Res. 2014;1587:88-96.

42. Yamamoto S, Kim HF, Hikosaka O. Reward value-contingent changes of visual responses in the primate caudate tail associated with a visuomotor skill. J Neurosci. 2013;33:11227-38.

43. Kim HF, Ghazizadeh A, Hikosaka O. Dopamine neurons encoding long-term memory of object value for habitual behavior. Cell. 2015;163:1165-75.

44. Anderson BA, Kuwabara H, Wong DF, Gean EG, Rahmim A, Brasic JR, et al. The role of dopamine in value-based attentional orienting. Curr Biol. 2016;26:1-6.

45. Hickey C, Chelazzi L, Theeuwes J. Reward changes salience in human vision via the anterior cingulate. J Neurosci. 2010;30:11096-103.

46. Goldstein RZ, Tomasi D, Alia-Klein N, Honorio Carrillo J, Maloney T, Woicik PA, et al. Dopaminergic response to drug words in cocaine addiction. J Neurosci. 2009;29:6001-6.

47. Schultz W. Updating dopamine reward signals. Curr Opin Neurobiol. 2013;23:229-38.

48. Elsworth JD, Jentsch JD, Morrow BA, Redmond DE Jr., Roth RH. Clozapine normalizes prefrontal cortex dopamine transmission in monkeys subchronically exposed to phencyclidine. Neuropsychopharmacology. 2008:33:491-6.

49. Porrino LJ, Goldman-Rakic PS. Brainstem innervation of prefrontal and anterior cingulate cortex in the rhesus monkey revealed by retrograde transport of HRP. J Comp Neurol. 1982;205:63-76.

Open Access This article is licensed under a Creative Commons Attribution-NonCommercial-ShareAlike 4.0 International License, which permits any non-commercial use, sharing, adaptation, distribution and reproduction in any medium or format, as long as you give appropriate credit to the original author(s) and the source, provide a link to the Creative Commons license, and indicate if changes were made. If you remix, transform, or build upon this article or a part thereof, you must distribute your contributions under the same license as the original. The images or other third party material in this article are included in the article's Creative Commons license, unless indicated otherwise in a credit line to the material. If material is not included in the article's Creative Commons license and your intended use is not permitted by statutory regulation or exceeds the permitted use, you will need to obtain permission directly from the copyright holder. To view a copy of this license, visit http://creativecommons.org/licenses/by-nc-sa/4.0/.

(c) The Author(s) 2019 